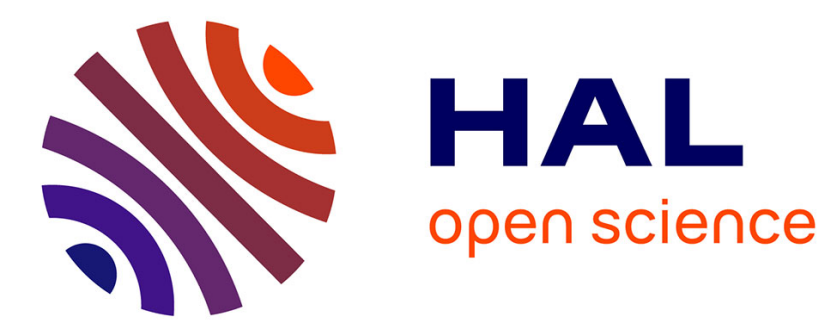

\title{
Covalent Chemistry for Graphene Electronics
}

Sandip Niyogi, Elena Bekyarova, Jeongmin Hong, Sakhrat Khizroev, Claire

Berger, Walt A. de Heer, Robert C. Haddon

\section{To cite this version:}

Sandip Niyogi, Elena Bekyarova, Jeongmin Hong, Sakhrat Khizroev, Claire Berger, et al.. Covalent Chemistry for Graphene Electronics. Journal of Physical Chemistry Letters, 2011, 2 (2487), pp.24872498. 10.1021/jz200426d. hal-01002907

\section{HAL Id: hal-01002907 https://hal.science/hal-01002907}

Submitted on 30 May 2020

HAL is a multi-disciplinary open access archive for the deposit and dissemination of scientific research documents, whether they are published or not. The documents may come from teaching and research institutions in France or abroad, or from public or private research centers.
L'archive ouverte pluridisciplinaire HAL, est destinée au dépôt et à la diffusion de documents scientifiques de niveau recherche, publiés ou non, émanant des établissements d'enseignement et de recherche français ou étrangers, des laboratoires publics ou privés. 


\section{Covalent Chemistry for Graphene Electronics}

Sandip Niyogi, ${ }^{+,}$Elena Bekyarova, ${ }^{+, \S}$ Jeongmin Hong, ${ }^{\perp}$ Sakhrat Khizroev, ${ }^{\perp, \S}$ Claire Berger, ${ }^{\#, \|}$ Walt de Heer," and Robert C. Haddon ${ }^{*,+,+, \S}$

${ }^{\dagger}$ Department of Chemistry, ${ }^{\ddagger}$ Department of Chemical \& Environmental Engineering, ${ }^{\S}$ Center for Nanoscale Science and Engineering, and ${ }^{\perp}$ Department of Electrical Engineering, University of California, Riverside, California 92521, United States

${ }^{\#}$ CNRS/Institut Néel, BP166, 38042 Grenoble, France

School of Physics, Georgia Institute of Technology, Atlanta, Georgia 30332, United States

The atomically flat surface of graphene provides an opportunity to apply carbon-carbon bond-forming chemical reactions to engineer the electronic properties of graphene circuitry. In particular, covalent functionalization of the surface or the edge of graphene ribbons provides a novel way to introduce patterning that can modulate the energy band gap, affect electron scattering, and direct current flow by producing dielectric regions in a graphene wafer. We discuss the use of Raman spectroscopy and scanning tunneling microscopy to characterize the surface functionalization periodicities and densities that have been produced by the chemical derivatization of epitaxial graphene together with the concomitant changes in the electronic and magnetic properties of the graphene surface layer.

The isolation of graphene supported on Si wafers $(\mathrm{XG}),{ }^{1}$ the synthesis of epitaxial graphene (EG), ${ }^{2}$ and the fabrication of graphene nanoribbons ${ }^{3,4}$ has now placed the graphene twodimensional $\pi$-electron system on the semiconductor industry roadmap for emerging logic devices. In principle, the availability of large-area graphene wafers offers the opportunity to fabricate the full complement of Si CMOS electronic devices and circuitry from carbon and additionally provides a route to new state variables for the semiconductor industry. Such an approach would require the atom-by-atom chemical functionalization of the whole graphene wafer and would therefore involve the practice of organic chemistry with a precision that is usually achieved only within natural systems. ${ }^{5}$ Chemically modified graphene is expected to be broadly useful, and applications in catalysis and energy are already the subject of article highlights. ${ }^{6,7}$ In the present Perspective, we discuss the use of Raman microscopy and spectroscopy, scanning tunneling microscopic imaging, and physical property measurements on nitrophenyl (NP)-functionalized EG as a rational methodology to advance the covalent chemistry of graphene for future electronic applications.

In the case of silicon CMOS technology, silicon dioxide served as the insulator for many years but is gradually being replaced by materials with higher dielectric constants (for the transistor gate, although the $\mathrm{Si} / \mathrm{SiO} 2$ interface is still present) and lower di-electric constants (for the interconnect insulation). The inter-connect wire is now copper after many years of utilizing alumi-num as the metallic conductor (no energy gap), and the logic devices are still field effect transistors based on doped silicon channel semiconductors (band gap $\approx 1.1 \mathrm{eV}$ ). There are many important engineering characteristics for these latter two materials, which include the mobility $(\mu)$, which is typically $\mu=43 \mathrm{~cm}^{2} / \mathrm{V}$ sec (copper) and $\mu$ (electron) $=280 \mathrm{~cm}^{2} / \mathrm{V} \mathrm{sec}$ (doped silicon), and the carrier concentration $(n)$, where $n=8.5 \times 10^{22} \mathrm{~cm}^{-3}$ (copper) and $n \approx 10^{20} \mathrm{~cm}^{-3}$ (dopant concentration) in transistors used today. 


\section{Energy band gap in metallic carbon nanotubes}

a)

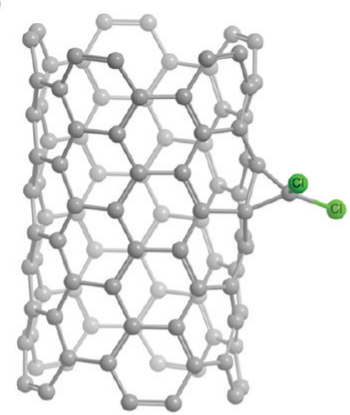

b)

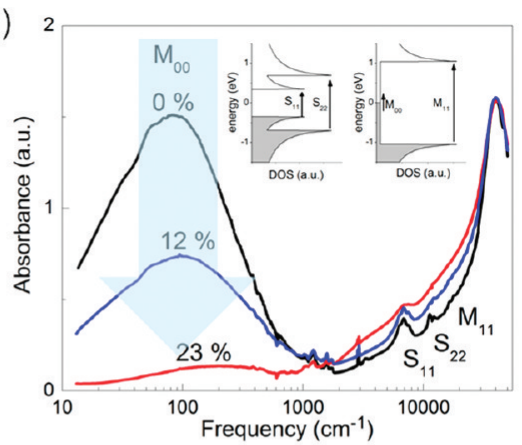

Energy band gap in epitaxial graphene

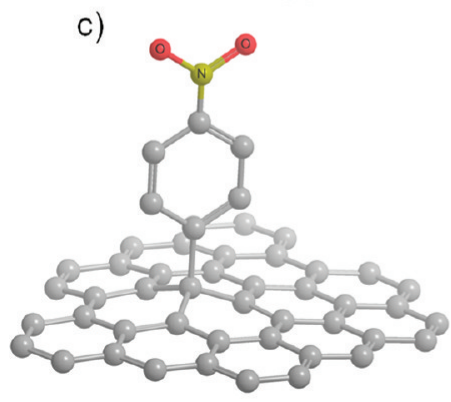

d)
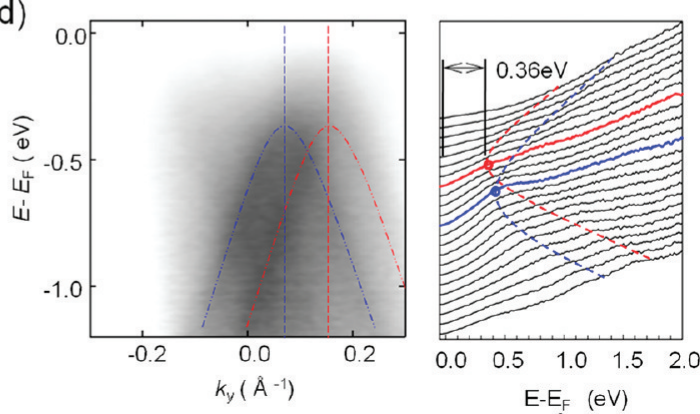

Figure 1. Energy band gap in carbon nanotubes and graphene induced by covalent chemical functionalization. (a) Structural schematic of a dichlorocarbene functionalized $(5,5)$ SWNTs. (b) Change in the optical absorption spectrum of SWNTs as a function of the density of side-wall functional groups. The absorption band in the far-IR region is characteristic of the metallic SWNTs and is lost as dichlorocarbene progressively adds across $\mathrm{C}=\mathrm{C}$ bonds and saturates the conjugation. ${ }^{13,14}$ The inset shows a schematic of the interband transitions in SWNTs. (c) Structural schematic of NP-functionalized graphene. (d) Angle-resolved photoelectron emission spectroscopy of NP-functionalized epitaxial graphene showing two diffuse bands highlighted by the dashed lines, corresponding to Dirac cones with a band edge $0.36 \mathrm{eV}$ below the Fermi level, estimated from constant energy cuts. ${ }^{15}$

In terms of the electrical characteristics of graphene, it has already been shown that graphane, $(\mathrm{CH})_{n}$, and fluorographene, $(\mathrm{CF})_{n}$, are highly resistive ${ }^{9-12}$ and thus could provide the dielectric and serve to pattern the wafer with insulating regions in order to inhibit the flow of an electrical current. The other key components are the logic devices and the wiring (interconnects). It is well-known that graphene can exhibit mobilities, $\mu>$ $200000 \mathrm{~cm}^{2} / \mathrm{V} \mathrm{sec}$, but at very low carrier densities, $n_{2 \mathrm{D}} \approx$ $10^{11} \mathrm{~cm}^{-2}\left(n_{3 \mathrm{D}} \approx 10^{19} \mathrm{~cm}^{-3}\right)$; furthermore, pristine graphene is a zero band bap semiconductor with a vanishingly small density of states at the Fermi level, even though it remains a conductor without the usual complement of carriers. Clearly, it represents an imposing chemical challenge to fabricate interconnects and logic devices in a wafer of graphene.

Beyond conventional Si CMOS components, logic devices based on new state variables, such as electronic and nuclear spin, pseudospin, and quantum interference, have been proposed for graphene. The development of these technologies requires very specific preparations of graphene. For example, graphene can act as a half-metallic ferromagnet when the current is allowed to pass along a zigzag edge. ${ }^{16}$ At a sharp $\mathrm{p}-\mathrm{n}$ junction (order of electron de Broglie wavelength) of graphene, it will be possible to focus, collimate, and observe lensing effects of electrons and build devices based on electron optics. ${ }^{17}$ An array of graphene quantum dots has been proposed for spin qubits. ${ }^{18}$ Lithographic fabrication of conducting channels of graphene in order to generate a confinement-related energy band gap may result in a high density of carbon dangling bonds, which imposes restrictions on the edge structure. ${ }^{19}$
Alternatively, patterning of the conjugated network could be achieved using $\mathrm{C}-\mathrm{C}$ bond transformation reactions, so that lattice vacancies or dangling bonds are eliminated. The covalent functionalization approach to band gap engineering of periodic conjugated carbon materials was first demonstrated in singlewalled carbon nanotubes (SWNTs); Figure 1 shows the progressive introduction of a band gap in metallic SWNTs as a function of the density of side-wall functionalization. The dichlorocarbene molecule used in this reaction forms a covalent bond across the double bonds, and as the number of functional groups increases, the conjugation length in the metallic SWNTs is reduced, and the electronic band structure reflects the removal of states at the Fermi level. ${ }^{14}$ It is possible to envisage a suite of chemical-bondforming reactions such that the conjugation of one, two, four, or all six $\pi$-electrons in the graphene six-membered ring are disrupted..$^{20,21}$

Electronic Structure of the Radical Addition Product of Graphene. The electronic and magnetic properties of graphene functionalized by radicals at single carbon centers have been studied theoretically, showing that it is possible to functionalize in a manner that gives rise to a band gap and under certain circumstances may lead to ferro- (ferri-) magnetism. ${ }^{16,22-29}$ Many of these theoretical treatments refer to hydrogen chemisorption defects that correspond to the addition of a hydrogen atom, but in terms of the electronic structure of the resulting $\pi$-radical, which becomes embedded in the graphene lattice, the aryl radical and hydrogen atom addition processes are functionally identical. A number of routes have been reported for the generation of aryl radicals, ${ }^{30}$ but the reduction of diazonium salts offers one of the 


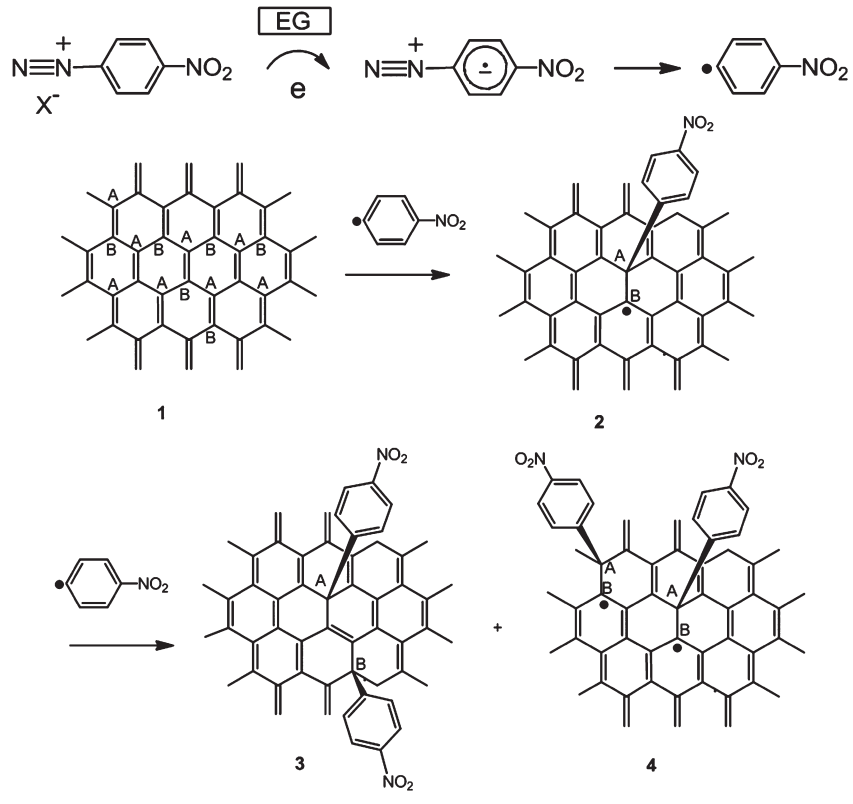

${ }^{a}$ The product structure represented in 3 is expected to be diamagnetic, while the structures represented in 4 would give rise to a ferromagnetic interaction between the spins. ${ }^{16,22-29}$

simplest mechanisms for grafting aryl groups to the surface of $\mathrm{sp}^{2}$-hybridized carbon materials, ${ }^{31-33}$ resulting in the generation of a delocalized $\pi$-radical (2, Scheme 1); although we display this as a single Kekule structure in Scheme 1, detailed calculations show that spin density is delocalized over many carbon atoms, with the highest spin density in the vicinity of the point of attachment of the aryl group (discussed later). ${ }^{22,23}$ For small substituents, pairwise additions in the $\mathrm{A}$ and $\mathrm{B}$ sublattices are favored on thermodynamic grounds, ${ }^{24,25}$ resulting in formally spinpaired Kekule products such as 3, although the preferred 1,4-mode of functionalization ( $25 \%$ coverage) is precluded by the physical size of the NP group. ${ }^{24,25,34}$ There are a number of additional factors that suggest that other modes of addition may be possible and that magnetic products such as $\mathbf{4}$ may be produced in which both additions have occurred in the $A$ sublattice, thereby leading to open-shell (non-Kekule) ${ }^{35}$ structures. ${ }^{22,23}$ The aryl radical is an extremely reactive species and the addition reactions may occur under kinetic rather than thermodynamic control, thereby leading to statistically significant quantities of products such as 4 . Furthermore, under certain reaction conditions, the diazonium reaction produces doping of the graphene substrate rather than aryl radical addition; ${ }^{36}$ this is a particularly important observation and is discussed further below.

Relationship of the Graphene Radical Addition Product to Molecular Odd Alternant Hydrocarbon $\pi$-Radicals. It is of interest to relate the electronic structure of the quasi-localized states that are encountered in the hydrogen chemisorption defect in graphene and graphite to the familiar $\pi$-radicals that occur in organic chemistry. Discernible perturbations to the electronic structure of graphite and graphene by the hydrogen chemisorption defects have been estimated to extend up to $10 \mathrm{~nm}$ from the site of hydrogen attachment, ${ }^{22,37-40}$ and thus, the resulting spin density
Scheme 1. Structures of the NP-Functionalized Epitaxial Graphene Products Formed by the Spontaneous Electron Transfer from EG to p-Nitrobenzene Diazonium Salt ${ }^{a}$

is delocalized over more than 10,000 carbon atoms. These remarkable structures correspond to extraordinarily large carbon $\pi$-radicals; the very efficient delocalization of the spin density may be responsible for their chemical stability, which allows them to exist in the atmosphere and to be observed by a number of techniques. ${ }^{41}$ In the graphite literature, these superlattices are referred to as defect states, but as we show below, they have a very interesting relationship to an important class of well-defined organic $\pi$-radicals, those that may be classified as odd alternant hydrocarbons (OAHs). ${ }^{42}$ Thus, in this section, we relate the radical addition product of the graphene lattice to its smaller (molecular) carbon $\pi$-radical counterparts in organic chemistry such as benzyl (5) and phenalenyl (6) (Scheme 2), which both belong to the class of radicals known as OAHs. It is particularly opportune to cast the problem in terms of $\mathrm{OAH} \pi$-radicals as it is possible to solve directly for the coefficients of the nonbonding molecular orbital (NBMO) of these radicals by inspection. ${ }^{42}$ The molecular NBMO is the counterpart of the quasi-localized states that lie at the Fermi level in the hydrogen chemisorption defects in graphite and graphene, and in both cases, it constitutes the spin-bearing orbital. Briefly, in order to solve for the coefficients of the NBMO, the carbon atoms are divided into starred and unstarred atoms, in analogy to the division between the A and B sublattices in the physics literature; in odd systems, the starred atoms are taken to be the most numerous. Within the simple tight-binding Huckel MO method, it may be shown that the coefficients of the unstarred atoms are 0 and that the sum of the NBMO coefficients of the nearest neighbors of any unstarred atom is also 0 , and thus, it is trivial to solve for the coefficients in a NBMO; ${ }^{42}$ the process is illustrated by application to $\mathbf{5}$ and $\mathbf{6}$ in Scheme 2 .

In the present connection, we note that the trigonal symmetry of the graphene superlattice and the structure of the radical 2 suggests the phenalenyl radical (6) as the primary building block; ${ }^{43}$ addition of a substituent $\mathrm{R}$ (where $\mathrm{R}=\mathrm{H}, \mathrm{Me}, \mathrm{C}_{6} \mathrm{H}_{4} \mathrm{NO}_{2}, \ldots$ ) to the central carbon leads to $9 \mathrm{HR}$-phenalene (7), which is known to be extremely unstable due to the presence of the [12] annulene conjugated system. Furthermore, 7 is no longer a simple radical, and thus, it is not an appropriate point of comparison. This point throws further light on the radical that exists in the graphene superlattice; there is effectively an odd carbon atom at the boundary of the delocalized $\pi$-radical structure that balances the loss of the carbon atom that becomes $\mathrm{sp}^{3}$ hybridized and is thereby removed from conjugation upon radical addition (otherwise, every radical addition would yield diamagnetic products). This recognition offers a strategy to generate radical structures from the 9HR-phenalene building block (7); we can add an exocyclic methylene fragment in order to generate an additional site of conjugation, giving an odd number of conjugated carbon atoms and thereby producing an $\mathrm{OAH} \pi$-radical, which provides a convenient route to obtain the coefficients of the spin-bearing 
Scheme 2. Model Molecules Related to the Quasi-localized $\pi$-Radical Defect State in Graphene Which Is Generated upon the $\sigma$-Bond Addition of Radicals ${ }^{a}$<smiles>[C+]c1ccccc1</smiles><smiles>C1=CC2=CC=CC3=CC=CC(=C1)[C-]23</smiles>

5<smiles>CCCc1ccccc1</smiles>

5

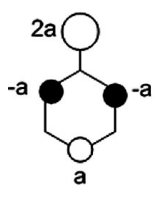

5, NBMO $a=7^{-1 / 2}$

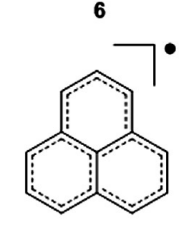

6

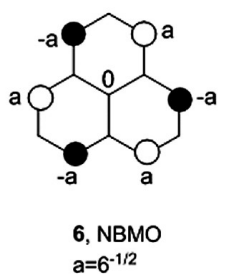<smiles>[R]C12C3=CC=CC1=CC=CC2=CC=C3</smiles>

7<smiles>[R]C12C=CC=C3C=CC=C1C=CC=C32</smiles><smiles>[R]C12C(=CC=CC3=CC(=C)C=C31)C=CC=C2C=CCC</smiles>

8

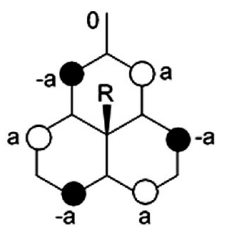

8, NBMO $a=6^{-1 / 2}$<smiles>[Z8]C1=C2C=CC=C3C=CC=C(C=C1)C32[R]</smiles><smiles>CC(C)=C1C=CC=C2C=CC=C3C=CCC(=C(C)C)C231</smiles>

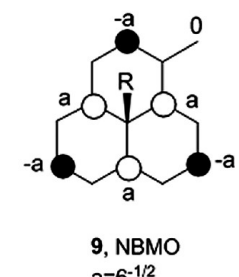

${ }^{a}$ Structures 5, 6, 8, and 9 show the Kekule and delocalized structures followed by the coefficients of the nonbonding molecular orbital (NBMO), which determine the spin densities at the level of simple HMO theory: the benzyl radical (5), the phenalenyl radical (6), 9HR-phenalene (7), 2-methylene9HR-phenalenyl (8), and 1-methylene-9HR-phenalenyl (9).

wave functions. There are two ways in which the methylene unit may be added to the periphery of 7, as shown in structures 8 and 9; in the case of $\mathbf{8}$, the resulting $\mathrm{NBMO}$ is identical to that of phenalenyl 6, whereas 9 leads to a rotation of the phenalenyl wave function by one carbon atom. In both cases, the coefficient (spin density) on the exocyclic methylene is 0 , and the resulting wave functions retains the trigonal symmetry of the graphene superlattice. As structure $\mathbf{9}$ is twice as prevalent as $\mathbf{8}$, it is to be expected that the coefficients in 9 will be the most important, although the coefficients are expected to drop off with distance from the site of functionalization. As discussed later, detailed calculations and STM experiments favor structure 9, although it should be noted that spin polarization effects lead to smaller (opposite) spin densities at the unstarred carbon atoms.

Thus the phenalenyl system (6-9) is identified as the building block for the radical addition product of graphene and this unit is readily extrapolated to structures of arbitrary size by fusion of rings of encircling carbon atoms (which preserve the threefold symmetry), followed by addition of a peripheral methylene group. Beyond phenalenyl ( $12+1$ conjugated carbons), the next member of the series is $\mathbf{1 0}(12+24+1$ conjugated carbon atoms).

It is important to note that radical structures such as $\mathbf{2}$ do not bring about a strong modification in the charge densities at the conjugated carbon atoms in the vicinity of the defect sites as the spin densities of such radicals are contained in NBMOs, and to first order, the charge densities are $0{ }^{42}$ The NBMOs necessarily lie at the Fermi level when embedded in the graphene lattice and bring about a strong modulation of the LDOS because of the confinement of the spin densities to either the A or B sublattices, and this may be visualized in the STM experiments (discussed later) as standing waves with peaks expected at the B sublattice and troughs in the A sublattice of 2 that extend up to $10 \mathrm{~nm}$ from the defect site. ${ }^{2,37,38,40}$
Scheme 3. Schematic Structure Showing the 4-NP Addition Product of a Finite Graphene Sheet and the Relationship of the A and B Sublattices to the Starred and Unstarred Atoms ${ }^{a}$

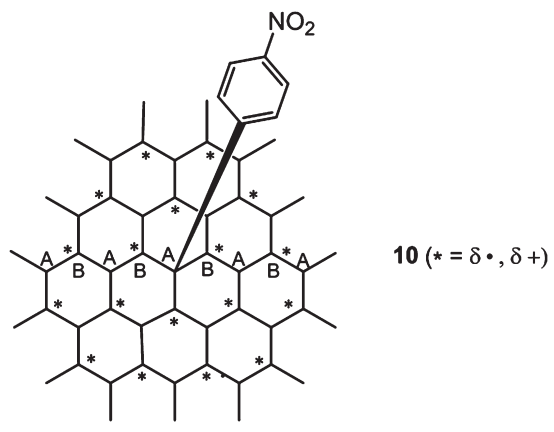

${ }^{a}$ Addition of the NP radical leads to spin densities at the starred atoms (no change in charge density), while the cation that is produced by oxidation (doping) of the radical replaces the spin densities with positive charge at the starred atoms.

As discussed above, under certain conditions, the diazonium reaction produces doping of the graphene substrate rather than aryl radical addition; ${ }^{36}$ doping of the graphene substrate is a natural consequence of the fact that the diazonium reaction requires an electron transfer from graphene in order to reduce the salt and initiate the reaction (Scheme 1). Given the fact that doping occurs simultaneously with aryl radical functionalization, it is important to consider how this will affect the subsequent chemistry. In fact, the easiest species to oxidize will be the $\pi$-radicals such as $\mathbf{2}$ as the NBMO is the highest (partially) occupied MO and lies at the Fermi level in the graphene radical addition product. As discussed above, the spin density in $\mathbf{2}$ is 
One and two graphene layers

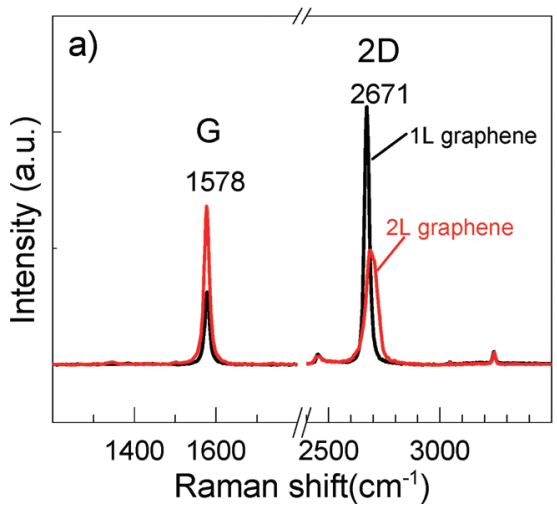

Functionalized epitaxial graphene

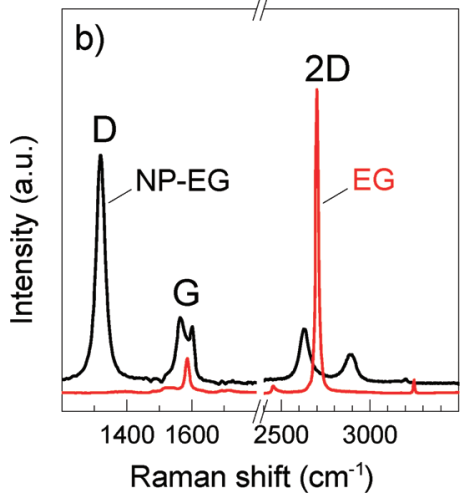

Functionalized

exfoliated graphene

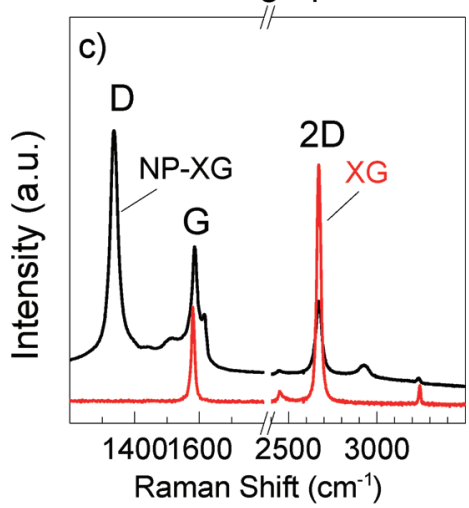

Figure 2. (a) Variation of Raman band intensities $\left(\lambda_{\mathrm{ex}}=532 \mathrm{~nm}\right)$ of graphene with the number of layers. Raman spectrum of exfoliated graphene and bilayer graphene, collected under identical laser power, spot size, and microscope aperture. The G band intensity of bilayer graphene is approximately twice that of a single layer of graphene. Raman spectral characteristics of covalently functionalized epitaxial graphene (b) and exfoliated graphene (c). The $\mathrm{SiC}$ signal has been subtracted from the epitaxial graphene spectra. After functionalization, the $\mathrm{D}$ band is the most intense peak, and the $2 \mathrm{D}$ band intensity decreases.

mainly confined to the NBMO, as illustrated in Scheme 3 (10), and if the addition occurs in the A sublattice, this places spin on the $\mathrm{B}$ sublattice (starred atoms in the $\mathrm{OAH}$ radical 10). However, if this radical $(\mathbf{2}, \mathbf{1 0})$ becomes oxidized to a cation by the doping process that is required for the initiation of the diazonium reaction, it is exactly the same set of B sublattice carbon atoms that now acquire a partial positive charge; under these conditions, it is possible that the reaction of the aryl radical with the $\mathrm{A}$ sublattice may be preferable, thereby leading to a situation in which functionalization occurs preferentially in a single sublattice as required for the occurrence of ferro- (ferri-) magnetism. ${ }^{22}$

Characterization of Covalently Functionalized Graphene. The Raman spectrum of single-layer graphene (1-LG) has two characteristic peaks, the $G$ peak at around $1580 \mathrm{~cm}^{-1}$ and the $2 \mathrm{D}$ peak (sometimes referred to as $\mathrm{G}^{\prime}$ ) at around $2670 \mathrm{~cm}^{-1}, 44,45$ and the intensity of the $2 \mathrm{D}$ peak is about three times the intensity of the $\mathrm{G}$ band (Figure 2a). The $\mathrm{G}$ band is a first-order Raman effect where the energy of the scattered incident monochromatic light is proportional to the energy of quantized lattice vibrations ( $\mathrm{E}_{2 \mathrm{~g}}$ phonon) generated as a result of the scattering process. The $2 \mathrm{D}$ band is a second-order Raman effect caused when the lattice vibrations due to the first-order process activate another phonon. In graphene, these three processes are in resonance, and in the Raman spectrum, the $2 \mathrm{D}$ band appears as a single peak of width $\sim 24 \mathrm{~cm}^{-1}$ and is greater in intensity than the $\mathrm{G}$ band. With increasing numbers of graphene layers in a sample, the $2 \mathrm{D}$ band can be resolved into multiple peaks at higher and lower energies, and the intensity decreases with respect to the $\mathrm{G}$ band intensity. For example, in bilayer graphene, the $2 \mathrm{D}$ band can be fit to four Lorentzians (Figure 2a). If the conjugation of the six-membered rings of graphene are disrupted by conversion of $\mathrm{sp}^{2}$ carbons to $\mathrm{sp}^{3}$ hybridization, the $\mathrm{A}_{1 \mathrm{~g}}$ mode breathing vibration of six-membered $\mathrm{sp}^{2}$ carbon rings is activated and is observed as the $\mathrm{D}$ band at $\sim 1340 \mathrm{~cm}^{-1}$. Intense $\mathrm{D}$ bands are observed in physically defective graphitic materials, graphene nanoribbons, and in disordered graphene samples. ${ }^{46}$

Covalent $\mathrm{C}-\mathrm{C}$ bond formation results in $\mathrm{sp}^{3}$ centers, and the Raman D band (Figure $2 \mathrm{~b}$ and $\mathrm{c}$ ) has been used to characterize covalent functionalization of graphene., $15,47-50$ The polarization dependence of the Raman spectra may provide additional resolution of the spatial distribution of $\mathrm{sp}^{3}$ centers. ${ }^{51}$ Additionally, those samples with a 2D band line width of $\sim 24 \mathrm{~cm}^{-1}$ and a larger size of graphene domain with identical 2D band characteristics show higher mobility. ${ }^{52}$ Thus, the Raman $\mathrm{D}$ and $2 \mathrm{D}$ band characteristics are a rapid analytical tool for the identification of functionalization in graphene as well as providing comparative information relevant to the transport properties of related samples.

However, there are a few experimental observations that are not completely understood and should be taken into account when analyzing the effects of chemical functionalization using Raman spectroscopy. In multilayer epitaxial graphene samples, a distribution of $2 \mathrm{D}$ band frequencies and line widths is observed (Figure 3a). Interfacial trapped charge, lateral strain, and stacking order of graphene layers have all been considered as plausible explanations of the observed $2 \mathrm{D}$ band characteristics. We have found that oxidatively etched as well as multilayered samples grown on conductive $\mathrm{SiC}$ wafers (doped) do not show such variations. $^{53}$

Transport in multilayered epitaxial graphene is further complicated by the presence of multiple conducting layers, whereas functionalization chemistry can only change the electronic properties of the surface layer. In exfoliated graphene samples, it has been shown that surface adsorbates, physical defects, and disorder lower the carrier mobility, ${ }^{19,54}$ whereas an increase in the dielectric constant of the medium in contact with the conducting graphene channel results in an increased mobility. ${ }^{55}$ The effects of ordered functionalization can be established by systematic studies of transport characteristics and atomically resolved imaging of microscopic devices. ${ }^{56}$ In bilayer and multilayer graphene samples, the $\mathrm{D}$ band intensity due to functionalization is typically weaker than that in a monolayer of graphene under similar reaction conditions, and this has been interpreted as a lowered reactivity of multilayered graphene as a result of interlayer electronic interaction. ${ }^{15,49}$ Direct imaging of the work function of multilayered epitaxial graphene indicates that single-layer graphene should be more reactive toward electron-transfer reactions as the graphene work function increases with the number of layers, although additional factors come into play such as the nature of the interface states at the $\mathrm{SiC}$ and the 


\section{D band of multilayer epitaxial graphene}

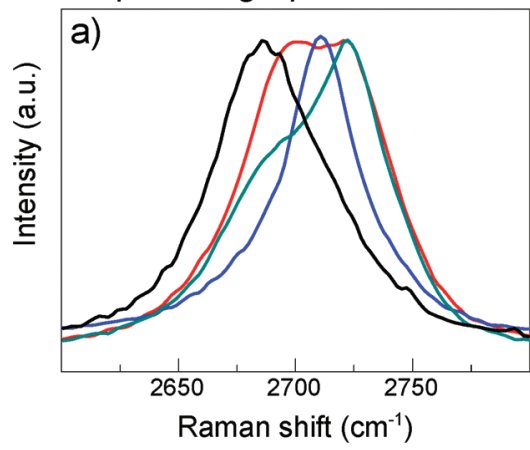

\section{Depth profile of epitaxial graphene and $\mathrm{SiC}$}

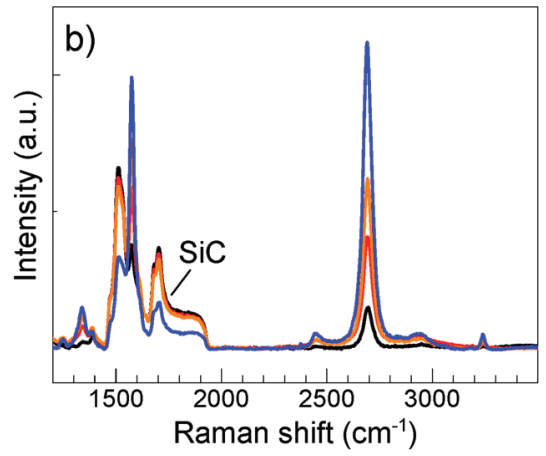

Depth profile after subtracting $\mathrm{SiC}$ signal

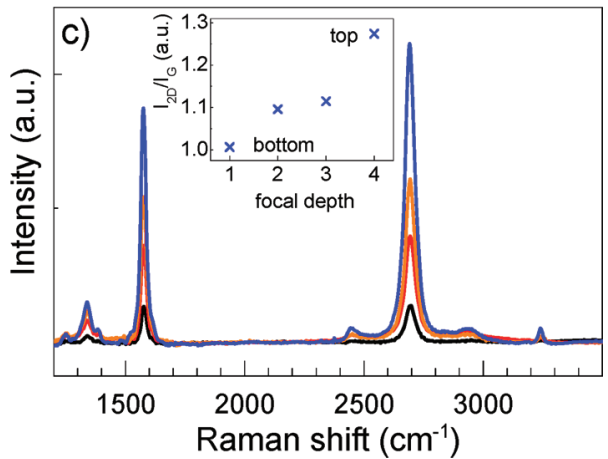

Figure 3. (a) Variation of the $2 \mathrm{D}$ peak frequency and line widths in multilayer epitaxial graphene. (b) Raman spectrum of multilayered epitaxial graphene on $4 \mathrm{H}-\mathrm{SiC}(0001)$ under identical spectrophotometer parameters but with changing focus, as indicated by the different intensities of the SiC emission lines between 1500 and $1900 \mathrm{~cm}^{-1}$. On the top of the wafer, the $\mathrm{SiC}$ signal is the weakest and increases as the focus is shifted lower. (c) Spectra of epitaxial graphene after subtracting the $\mathrm{SiC}$ signal. The inset shows that the $I_{2 \mathrm{D}} / I_{\mathrm{G}}$ ratio increases as the focus is shifted to the top of the sample, indicating a decrease in the interlayer electronic coupling.

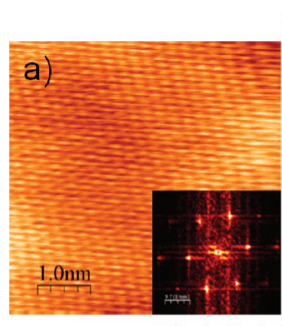

Epitaxial graphene
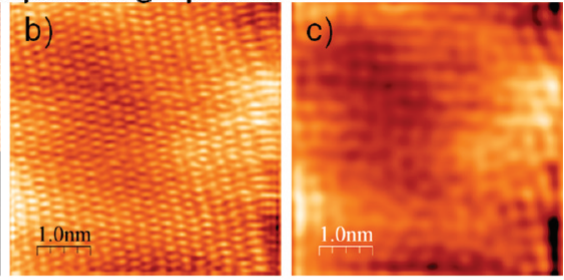

Point defects in epitaxial graphene
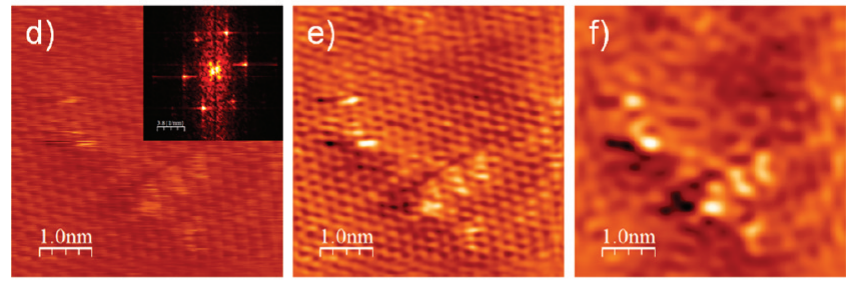

Nitrophenyl functionalized epitaxial graphene
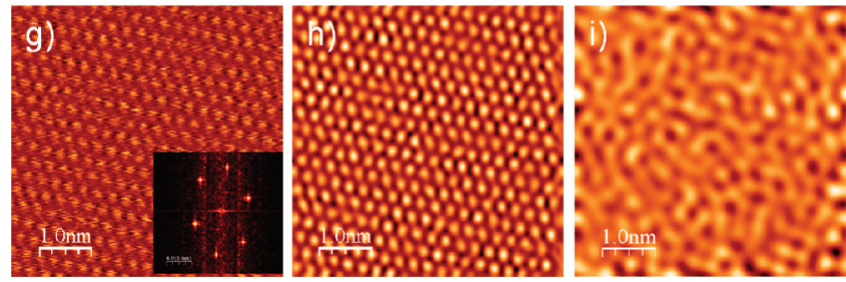

Figure 4. STM image of epitaxial graphene under ambient conditions. (a) Pristine EG, $V_{\text {bias }}=+5.1 \mathrm{mV}, I_{\mathrm{t}}=4.3 \mathrm{nA}$; a 2D-FFT spectrum of the STM image is shown in the inset. (b) STM image after subtracting noise. (c) 2D-FFT filtered STM image. (d) Pristine EG with defects, $V_{\text {bias }}=+1$ $\mathrm{mV}, I_{\mathrm{t}}=9.4 \mathrm{nA}$; a 2D-FFT spectrum of the STM image is shown in the inset. (e) STM image after subtracting noise. (f) 2D-FFT filtered STM image. (g) NP-functionalized EG, $V_{\text {bias }}=+1.1 \mathrm{mV}, I_{\mathrm{t}}=5.3 \mathrm{nA}$; a 2D-FFT spectrum of the STM image is shown in the inset. (h) STM image after subtracting noise. (i) 2D-FFT filtered STM image.

presence of trapped charge. ${ }^{57}$ However, Raman spectroscopy is not a surface-sensitive technique and as shown in Figures $3 b, c$ and $2 a$, the intensity of the $G$ band of both exfoliated and epitaxial graphene clearly scales with the number of graphene layers. Thus, a similar density of $\mathrm{sp}^{3}$ centers on the surface of graphite would result in a weaker $D$ band intensity relative to the $G$ band, compared to graphene.

Scanning tunneling microscopic (STM) imaging can be used to directly determine the surface coverage of functional groups on the graphene surface; imaging covalently attached molecules typically requires ultrahigh vacuum and cryogenic temperatures. However, it has been shown both theoretically and experimentally that the local electronic structure at isolated defect sites in both graphite and graphene generate three-fold symmetric patterns, particularly after the introduction of hydrogen chemisorption sites by proton irradiation..$^{22,37-40}$ These patterns can be enhanced by two-dimensional fast Fourier transform (2D-FFT) filtering of STM images acquired under ambient conditions, and it has been shown that a defect on the A or B sublattices in graphite generates distinguishable three-fold-symmetric patterns. ${ }^{37,38}$ The hydrogen chemisorption defect generates the same electronic structure in the graphene lattice as NP functionalization (see 2, Scheme 1 and 10, Scheme 3), for which subsequent radical additions can give products such as $\mathbf{3}$ and $\mathbf{4}$. Both $\mathbf{2}$ and $\mathbf{4}$ are expected to be prominent in STM experiments because the resulting $\pi$-radical species give rise to half-filled quasi-localized states that lie close to the Fermi level and thus will figure prominently in the local density of states (LDOS) near the site of functionalization; this allows the direct observation of the $\pi$-radical wave functions discussed above.

In Figure 4, STM images of epitaxial graphene and NPfunctionalized epitaxial graphene are compared; the STM images were acquired using a Digital Instruments Nanoscope IIIa multimode scanning probe microscope under ambient conditions, with commercial Pt/Ir tips, and 2D-FFT filtering was performed using the WSxM package. ${ }^{58}$ The 2D FFT spectrum of a STM image of graphene consists of the six outer bright spots from the graphene superlattice and six spots corresponding to the graphene lattice in the center, appearing as a larger bright spot in the insets of Figure $4 \mathrm{a}, \mathrm{d}$, and g. Filtering the higher-order spots in the FFT spectrum (not shown) removes the noise in the STM images (Figure $4 b$, e, and h), while filtering the graphene lattice by removing everything inside of the largest circle circumscribed by the hexagon of the supperlattice points yields Figure $4 c$, $\mathrm{f}$, and i, which show the modified LDOS. ${ }^{22,37-40}$ Figure 4a is a STM 


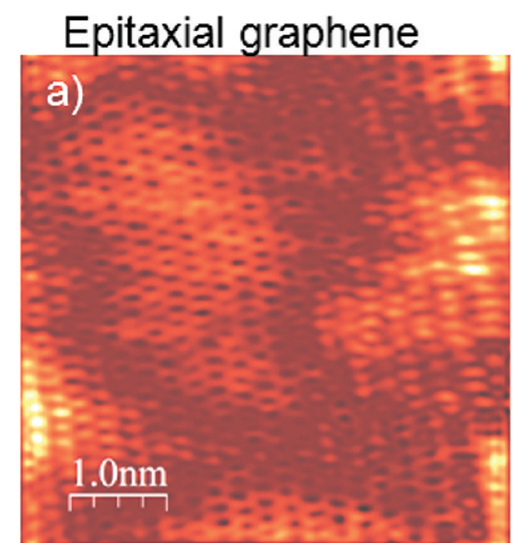

c)



NP-functionalized EG

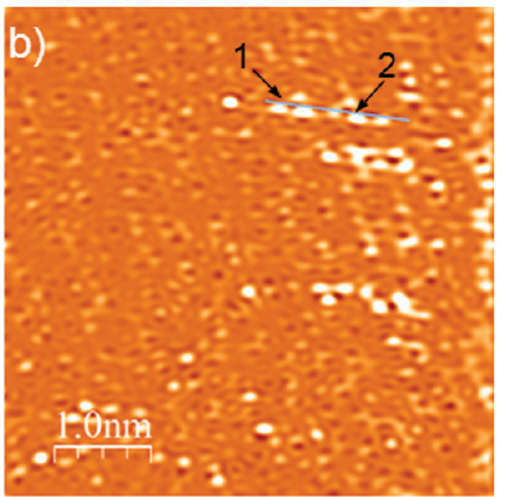

d)

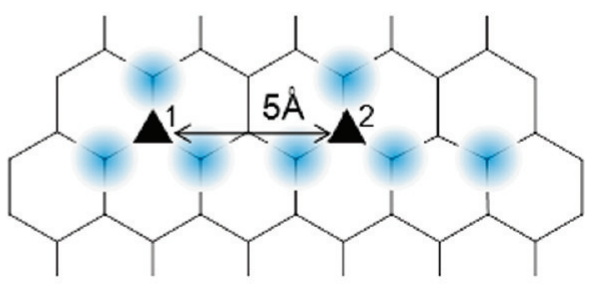

Figure 5. Superimposed STM images and FFT filtered-STM images. (a) Figure 4c superimposed in Figure 4b. (b) Figure 4i superimposed in Figure 4h. (c) Line scan of panel b, where the LDOS is dominated by superstructures. (d) Schematic representation of atoms labeled 1 and 2 in panel b. The distance is measured from the STM line scan shown in panel c.

a) Pristine


b) NP functionalized

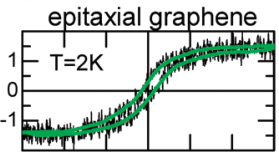

$\begin{array}{lllll}-2 & -1 & 0 & 1 & 2\end{array}$
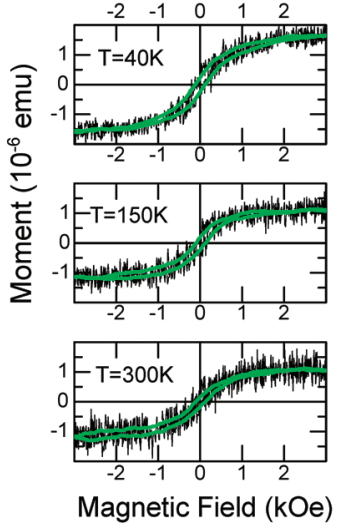

c) NP functionalized top graphene layer
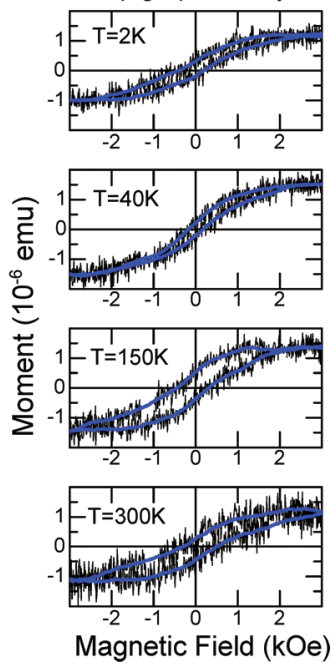

Figure 6. In-plane magnetization measured at different temperatures of pristine EG on SiC (a) and NP-functionalized EG (b) and the magnetism of EG due to NP functionalization (c) obtained by numerically subtracting the data of pristine EG from that of NP-functionalized EG. The diamagnetism of SiC is subtracted from the recorded signal of the pristine and functionalized EG samples. ${ }^{41}$

image of a defect-free region of $1-3$ layer epitaxial graphene, and in Figure 4b, the bright and dark regions have been previously interpreted as being due to interference of electronic waves in graphene, forming a Moiré pattern. ${ }^{59,60}$ After 2D-FFT filtering
(Figure 4c), the peaks in the Fermi level LDOS are localized on the atomic lattice. The presence of point defects can be recognized in the STM topographic images (Figure 4d and e), which generates an isolated three-fold symmetric pattern (Figure 4f). 

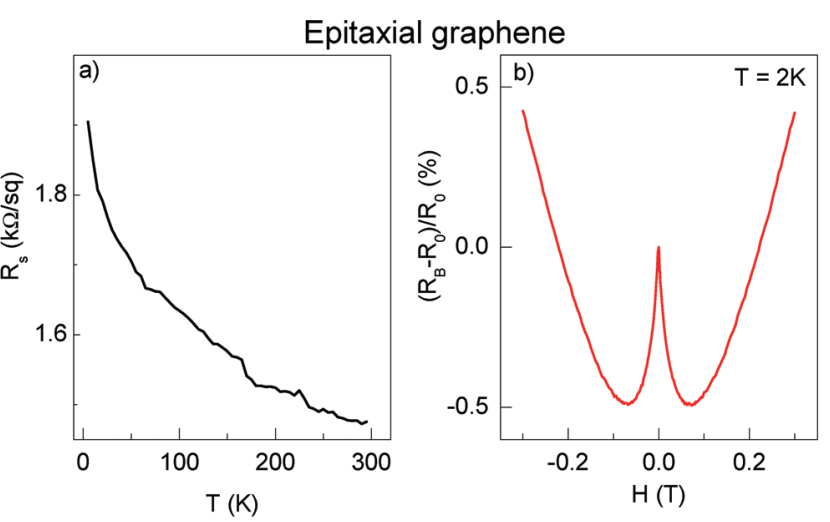

Nitrophenyl functionalized epitaxial graphene

The sample imaged in Figure $4 \mathrm{~g}$ has 3-5 layers of epitaxial graphene, of which the top surface was functionalized with NP groups, and the lattice topography does not show any defects (Figure 4h). However, the 2D-FFT filtered charge density (Figure 4i) shows scattering and interference patterns over the entire image.

Superimposing the FFT filtered image on the topographic image allows the visualization of the positions of the $\mathrm{sp}^{3}$ centers. $^{38}$ In Figure 5a, the images from defect-free graphene (Figure $4 \mathrm{~b}$ and c) are superimposed, and the image can be interpreted as an enhancement of the Moiré pattern observed in the topographic image. However, upon superimposing Figure $4 \mathrm{~h}$ and i, an irregular distribution of three- and six-fold symmetric patterns is observed (Figure $5 b$ ), with a preponderance of bright spots in a triangular geometrical arrangement that shows a striking correspondence with the NBMO coefficients of structure 9 in the immediate vicinity of the point of addition. Related patterns have been reported in the STM image of hydrogen-functionalized graphene on Ir (111). ${ }^{12}$ Sectional analysis (Figure 5c) shows that at the highest density region (two neighboring three-fold symmetric bright spots with a dark center), the distance between the dark centers is $0.54 \mathrm{~nm}$; a structural representation of the $\mathrm{sp}^{3}$ centers at this density is shown in Figure 5d. This corresponds to a functionalization event in which both of the NP $\mathrm{sp}^{3} \mathrm{C}$-centers are in the same sublattice of graphene, which is expected to give rise to ferromagnetic coupling between the spins. ${ }^{22,41}$ Atomically resolved STM imaging under ambient conditions makes this method particularly attractive for correlating the functional group density and periodicity in microscopic devices, where the transport characteristics can also be measured.

Electronic and Magnetic Properties of Covalently Functionalized Graphene. The formation of a covalent $\sigma$-bond to one of the basal plane graphene carbon atoms by attachment of a radical species such as a hydrogen atom or the spin-bearing carbon atom of an organic molecule necessarily generates a delocalized spin in the graphene $\pi$ system, and if subsequent functionalization takes place in the same sublattice (above), an open-shell structure results. The long-range coupling necessary for magnetic ordering of the local moments at room temperature in the semiconducting host is expected to take place through spin alternation due to the presence of half-filled $\pi$ orbitals in the $\mathrm{sp}^{2}$-bonded bipartite lattice of graphene. ${ }^{23,61-63} \mathrm{In}$ order to investigate the change in the magnetic properties of epitaxial graphene as a result of chemical functionalization, we subtracted the $\mathrm{M}-\mathrm{H}$ data from measurements carried out on the same sample, before and after chemical reaction, thereby eliminating the contributions of the $\mathrm{SiC}$ substrate and the pristine epitaxial
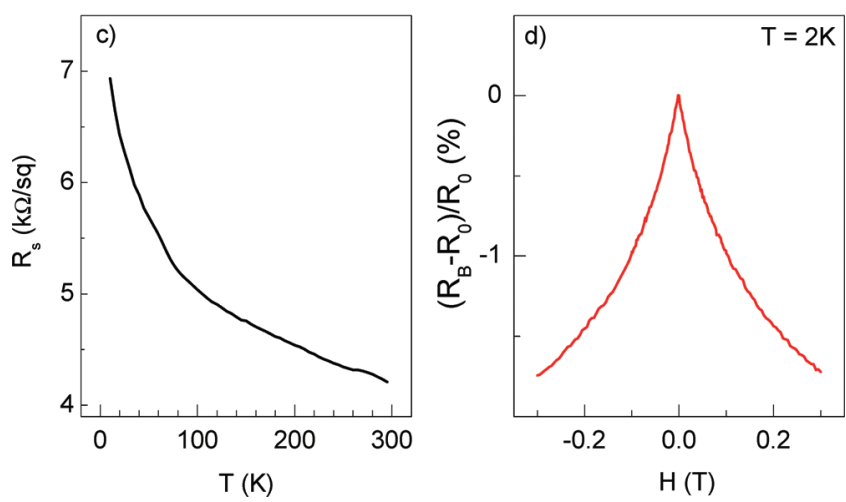

Figure 7. (a) Sheet resistance as a function of temperature and (b) outof-plane magnetoresistance at $T=2 \mathrm{~K}$ of epitaxial graphene. (c) Sheet resistance and (d) out-of-plane magnetoresistance of NP-functionalized epitaxial graphene at $T=2 \mathrm{~K}$.

graphene sublayers. Figure 6 shows the magnetization of a representative EG sample in the pristine state and after functionalization as a function of the magnetic field at different temperatures.

A small magnetization is observed in the pristine sample at all temperatures, which may be due to defects in the SiC crystal lattice or in the epitaxial graphene layers. The difference in the $\mathrm{M}-\mathrm{H}$ data between the pristine (Figure 6a) and the NP-functionalized sample (Figure 6b) is due to the change in the magnetic properties of the top layer of graphene as a result of NP functionalization (Figure 6c), and it shows a nonlinear dependence on a magnetic field strength and a clear hysteresis. Both the coercivity and the saturation magnetization vary with temperature and are sample-dependent. ${ }^{41}$

Magnetic, atomic, and electrostatic force microscopy were used to show that the surface of the pristine EG samples is inhomogeneous over varying length scales with respect to the number and stacking of graphene layers, crystalline domain size, trapped charge at the interfacial layer, as well as compressive strain and ridges. ${ }^{41}$ The data indicate that the magnetism is confined to clusters on the surface of the EG with varying values of the coercive field and is indicative of a distribution in the sizes and interaction strengths of the magnetic regions, which is typical of magnetic objects that are close to the border of ferromagnetism (ferrimagnetism) and superparamagnetism.

The change of resistance of epitaxial graphene as a function of temperature shows ideal semimetallic behavior with zero or small energy gap. In Figure 7a, the characteristics of a 5-7 layer epitaxial graphene sample $(3 \mathrm{~mm} \times 4 \mathrm{~mm})$ is shown; the increase 
in resistance with decreasing temperature is attributed to the decreasing carrier density and has been previously observed in graphite samples less than $10 \mathrm{~nm}$ thick. ${ }^{64-66}$ After NP functionalization, the room-temperature resistance of the sample increased from 0.4 to $3.2 \mathrm{k} \Omega$, and the semiconducting nature of the sample is supported by the observation of a $0.4 \mathrm{eV}$ band gap in ARPES measurements (Figure 1).

The resistance of the pristine EG sample as a function of magnetic field is shown in Figure $7 \mathrm{~b}$, and two distinct regimes can be identified when the field is perpendicular to the plane of the graphene wafer. In the low-field region $(|\mathbf{H}|<0.1 \mathrm{~T})$, the magnetoresistance (MR) is negative, whereas in the high-field region $(|\mathrm{H}|>0.1 \mathrm{~T})$, it becomes positive; the low-field negative $\mathrm{MR}$ is due to weak localization (WL), which occurs as a result of point defects that locally break the sublattice degeneracy, thereby causing intervalley scattering between the Dirac cones. At low temperatures, the electrons remain coherent over long distances as inelastic scattering does not destroy the phase of the electron waves. Thus, when two electrons start from the same point, travel in opposite directions in a closed loop, and meet at the point of origin, there is constructive interference because their phases remain intact, thereby increasing the probability of backscattering, which produces an increase in the electrical resistance. The presence of a magnetic field applied perpendicular to the plane of graphene disrupts the phases of the two electrons and is observed as negative magnetoresistance at low fields (WL).

Weak antilocalization (WAL) arises from long-range scattering, which does not distinguish between the $\mathrm{A}$ and the $\mathrm{B}$ atoms. Because the charge carriers in pristine EG are chiral, the backscattering is suppressed; however, the magnetic field destroys the equivalence between the A and B sublattices, and this suppression of the WAL by the magnetic field leads to a positive magnetoresistance. The NP functionalization also destroys the symmetry of the graphene sublattices, thereby quenching the WAL and leading to negative magnetoresistance over the entire magnetic field range (Figure 7c,d). ${ }^{67-69}$

Thus, aryl radical functionalization of epitaxial graphene not only provides a route for band gap engineering of graphene $e^{15,20,34}$ but leads to disordered magnetism in the graphene sheet that includes antiferromagnetic regions mixed with superparamagnetic and ferromagnetic (ferrimagnetic) clusters. If it were possible to optimize and control the chemical functionalization of the EG wafers so as to achieve periodic structures, it may be possible to induce long-range ferromagnetic order combined with periodic semiconductors, thereby leading to advanced spintronic devices. ${ }^{63,70-72}$ Previous experimental and theoretical investigations have referred to defective structures in achieving ferromagnetism in graphite and graphene; while functionally similar, the covalent functionalization approach does not generate physical defects in the graphene lattice, but rather, it leads to carboncarbon bonds and in some cases unpaired spins in a chemical approach to engineering electronic and magnetic devices such as ferromagnetic semiconductors in an organic macromolecule.

Chemistry will play a significant role in tailoring the electronic structure of graphene wafers for the generation of devices and circuitry. The investigation of NP functionalization of graphene provides a simple proof of concept for the prospects of covalent chemistry in graphene electronics, photonics, and spintronics and has allowed us to identify some of the characterization methods necessary for such research. The fundamental concept is the generation of new $\mathrm{C}-\mathrm{C}$ bonds to redirect the electronic conjugation pathway and to form specific conducting, insulating, and magnetic patterns. While the ultimate goals are to identify functionalization periodicities and $\mathrm{C}-\mathrm{C}$ bond hapticities to maintain the highest mobilities and to tune band gap energies, future advances in the experimental research will require a more detailed understanding of the morphology and physical properties of graphene grown on dimensions suitable for macroscopic characterization. The effects of heterogeneity in epitaxial graphene samples on chemical reactivity have been particularly important in the investigation of the magnetic and electronic properties of epitaxial graphene covalently functionalized with aryl radicals. Besides morphology, both epitaxial and CVD grown graphene have domain boundaries with 5-7-membered ring defects, ${ }^{73,74}$ and charge and spin transport across such boundaries have unique characteristics. ${ }^{75}$ The chemical reactivity of such defects can be expected to be different from that of periodic six-membered rings. The identification of the transport characteristics of covalently functionalized multilayer epitaxial graphene is further complicated by transport through underlying, pristine graphene layers ${ }^{53}$ and transport characteristics across $\mathrm{SiC}$ step edges ${ }^{76}$ and materials integration issues. ${ }^{77}$ The demonstration of the use of STM methods to image functionalization density and chemical structure, ${ }^{78}$ enabled by the atomically flat conducting surface of graphene, points the way for further research into the functionality and device chemistry of graphene. 


\section{ACKNOWLEDGMENT}

We acknowledge financial support from DOD/DMEA under Contract H94003-09-2-0901 and H94003-09-2-1003 and NSFMRSEC through Contract DMR-0820382.

\section{REFERENCES}

(1) Novoselov, K. S.; Geim, A. K.; Morozov, S. V.; Jiang, D.; Katsnelson, M. I.; Grigorieva, I. V.; Dubonos, S. V; Firsov, A. A. TwoDimensional Gas of Massless Dirac Fermions in Graphene. Nature 2005, 438, 197-200.

(2) Berger, C.; Song, Z.; Li, T.; Li, X.; Ogbazghi, A. Y.; Feng, R.; Dai, Z.; Marchenkov, A. N.; Conrad, E. H.; First, P. N.; et al. Ultrathin Epitaxial Graphite: 2D Electron Gas Properties and a Route Toward Graphene-Based Nanoelectronics. J. Phys. Chem. B 2004, 108, 19912-19916.

(3) Han, M. Y.; Ozyilmaz, B.; Zhang, Y. B; Kim, P. Energy Band-Gap Engineering of Graphene Nanoribbons. Phys. Rev. Lett. 2007, 98, 206805/1-206805/4.

(4) Sprinkle, M.; Ruan, M.; Hu, Y.; Hankinson, J.; Rubio-Roy, M.; Zhang, B.; Wu, X.; Berger, C; de Heer, W. A. Scalable Templated Growth of Graphene Naoribbons on SiC. Nat. Nanotechnol. 2010, $5,727-731$.

(5) Haddon, R. C; Lamola, A. A. The Molecular Electronic Device and the Biochip Computer: Present Status. Proc. Natl. Acad. Sci. U.S.A. 1985, 82, 1874.

(6) Kamat, P. V. Graphene-Based Nanoarchitectures. Anchoring Semiconductor and Metal Nanoparticles on a Two-Dimensional Carbon Support. J. Phys. Chem. Lett. 2010, 1, 520-527.

(7) Kamat, P. V. Graphene-Based Nanoassemblies for Energy Conversion. J. Chem. Phys. Lett. 2011, 2, 242-251.

(8) Nishi, Y.; Doering, R. Handbook of Semiconductor Manufacturing Technology; Marcel Dekker, Inc.: New York, 2000.

(9) Elias, D. C.; Nair, R. R.; Mohiuddin, T. M. G.; Morozov, S. V. B., P.; Halsall, M. P.; Ferrari, A. C.; Boukhvalov, D. W.; Katsnelson, M. I.; Geim, A. K; Novoselov, K. S. Control of Graphene's Properties by Reversible Hydrogenation: Evidence for Graphane. Science 2009, 323, 610-613.

(10) Jeon, K. J.; Lee, Z.; Pollak, E.; Moreschini, L.; Bostwick, A.; Park, C. M.; Mendelsberg, R.; Radmilovic, V.; Kostecki, R.; Richardson, T. J.; et al. Fluorographene: A Wide Bandgap Semiconductor with Ultraviolet Luminescence. ACS Nano 2011, 5, 1042-1046.

(11) Nair, R. R.; Ren, W.; Jalil, R.; Riaz, I.; Kravets, V. G.; Britnell, L.; Blake, P.; Schedin, F.; Mayorov, A. S.; Yuan, S.; et al. Fluorographene: A Two-Dimensional Counterpart of Teflon. Small 2010, 6, 2877-2884.

(12) Balog, R.; Jorgensen, B.; Nilsson, L.; Andersen, M.; Rienks, E.; Bianchi, M.; Fanetti, M.; Laegsgaard, E.; Baraldi, A.; Lizzit, S.; et al. Bandgap Opening in Graphene Induced by Patterned Hydrogen Adsorption. Nat. Mater. 2010, 9, 315-319.

(13) Kamaras, K.; Itkis, M. E.; Hu, H.; Zhao, B; Haddon, R. C. Covalent Bond Formation to a Carbon Nanotube Metal. Science 2003, $301,1501$.

(14) Hu, H.; Zhao, B.; Hamon, M. A.; Kamaras, K.; Itkis, M. E; Haddon, R. C. Sidewall Functionalization of Single-Walled Carbon Nanotubes by Addition of Dichlorocarbene. J. Am. Chem. Soc. 2003, 125, 14893-14900.

(15) Niyogi, S.; Bekyarova, E.; Itkis, M. E.; Zhang, H.; Shepperd, K.; Hick, J.; Sprinkle, M.; Berger, C.; Lau, C. N.; de Heer, W. A.; et al. Spectroscopy of Covalently Functionalized Graphene. Nano Lett. 2010, 10, 4061-4066.

(16) Son, Y.-W.; Cohen, M. L; Louie, S. G. Half-Metallic Graphene Nanoribbons. Nature (London) 2008, 444, 347-349.
(17) Cheianov, V. V.; Falko, V; Altshuler, B. L. The Focussing of Electron Flow and a Veselago Lens in Graphene $\mathrm{p}-\mathrm{n}$ Junctions. Science 2007, 315, 1252-1255.

(18) Trauzettel, B.; Bulaev, D. V.; Loss, D; Burkar, G. Spin Qubits in Graphene Quantum Dots. Nat. Phys. 2007, 3, 192-196.

(19) Wang, X. R.; Ouyang, Y.J.; Li, X. L.; Wang, H. L.; Guo, J; Dai, H.J. Room-Temperature All-Semiconducting Sub-10-nm Graphene Nanoribbon Field-Effect Transistors. Phys. Rev. Lett. 2008, 100, 206803-4.

(20) Bekyarova, E.; Itkis, M. E.; Ramesh, P.; Berger, C.; Sprinkle, M.; de Heer, W. A; Haddon, R. C. Chemical Modification of Epitaxial Graphene: Spontaneous Grafting of Aryl Groups. J. Am. Chem. Soc. 2009, 131, 1336-1337.

(21) Sarkar, S.; Bekyarova, E.; Niyogi, S; Haddon, R. C. Diels-Alder Chemistry of Graphite and Graphene: Graphene as Diene and Dienophile. J. Am. Chem. Soc. 2011, 133, 3324-3327.

(22) Yazyev, O. V. Emergence of Magnetism in Graphene Materials and Nanostructures. Rep. Prog. Phys. 2010, 73, 056501.

(23) Yazyev, O. V; Helm, L. Defect-Induced Magnetism in Graphene. Phys Rev. B 2007, 75, 125408.

(24) Boukhvalov, D. W; Katsnelson, M. I. Tuning the Gap in Bilayer Graphene using Chemical Functionalization: Density Functional Calculations. Phys. Rev. B 2008, 78, 085413.

(25) Jiang, D.; Sumpter, B. G; Dai, S. How Do Aryl Groups Attach to a Graphene Sheet?. J. Phys. Chem. B 2006, 110, 23628-23632.

(26) Fujita, M.; Wakabayashi, K.; Nakada, K; Kusakabe, K. Peculiar Localized State at Zigzag Graphite Edge. J. Phys. Soc. Jpn. 1996, 65, 1920-1923.

(27) Nakada, K.; Fujita, M.; Dresselhaus, G; Dresselhaus, M. S. Edge State in Graphene Ribbons: Nanometer Size Effect and Edge State Dependence. Phys Rev. B 1996, 54, 17954-17961.

(28) Wakabayashi, K.; Fujita, M.; Ajiki, H; Sigrist, M. Electronic and Magnetic Properties of Nanographite Ribbons. Phys Rev. B 1999, 59, $8271-8282$.

(29) Miyamoto, Y.; Nakada, K; Fujita, M. First-Principles Study of Edge States of H-Terminated Graphitic Ribbons. Phys Rev. B 1999, 59, 9858-9861.

(30) Liu, H.; Ryu, S.; Chen, Z.; Steigerwald, M. L.; Nuckolls, C; Brus, L. E. Photochemical Reactivity of Graphene. J. Am. Chem. Soc. 2009, 131, 17099-17101.

(31) Delamar, M.; Hitmi, R.; Pinson, J; Saveant, J. M. Covalent Modification of Carbon Surfaces by Grafting of Functionalized Aryl Radicals Produced from Electrochemical Reduction of Diazonium Salts. J. Am. Chem. Soc. 1992, 114, 5883-5884.

(32) Liu, Y. C; Mccreery, R. L. Reactions of Organic Monolayers on Carbon Surfaces Observed with Unenhanced Raman Spectroscopy. J. Am. Chem. Soc. 1995, 117, 11254-11259.

(33) Bahr, J. L.; Yang, J.; Kosynkin, D. V.; Bronikowski, M. J.; Smalley, R. E; Tour, J. M. Functionalization of Carbon Nanotubes by Electrochemical Reduction of Aryl Diazonium Salts: A Bucky Paper Electrode. J. Am. Chem. Soc. 2001, 123, 6536-6542.

(34) Bekyarova, E.; Itkis, M. E.; Ramesh, P; Haddon, R. C. Chemical Approach to the Realization of Electronic Devices in Epitaxial Graphene. Phys. Status Solidi RRL 2009, 3, 184-186.

(35) Borden, W. T.; Iwamura, H; Berson, J. A. Violations of Hund's Rule in Non-Kekule Hydrocarbons: Theoretical Prediction and Experimental Verification. Acc. Chem. Res. 1994, 27, 109-116.

(36) Farmer, D. B.; Golizadeh-Mojarad, R.; Perebeinos, V.; Lin, Y.-M.; Tulevski, G. S.; Tsang, J. C; Avouris, P. Chemical Doping and 
Electron-Hole Conduction Asymmetry in Graphene Devices. Nano Lett. 2009, 9, 388-392.

(37) Mizes, H. A.; Park, S; Harrison, W. A. Multiple-Tip INterpretation of Anoalous Scanning-Tunneling-Microscopy Images of Layered Materials. Phys. Rev. B 1987, 36, 4491-4494.

(38) Kelly, K. F; Halas, N. J. Determination of $\alpha$ and $\beta$ Site Defects on Graphite Using C60-Adsorbed STM Tips. Surf. Sci. 1998, 416, L1085-L1089.

(39) Ruffieux, P.; Groning, O.; Schwaller, P.; Schlapbach, L; Groning, P. Hydrogen Atoms Cause Long-Range Electronic Effects on Graphite. Phys. Rev. Lett. 2000, 84, 4910-4913.

(40) Ruffieux, P.; Melle-Franco, M.; Groning, O.; Bielmann, M.; Zerbetto, F; Groning, P. Charge-Density Oscillation on Graphite Induced by the Interference of Electron Waves. Phys. Rev. B 2005, 71, 153403.

(41) Hong, J.; Niyogi, S.; Bekyarova, E.; Itkis, M. E.; Palanisamy, R.; Amos, N.; Litvinov, D.; Berger, C.; de Heer, W. A.; Khizroev, S.; et al. Effect of Nitrophenyl Functinalization on the Magnetic Properties of Epitxaial Graphene. Small 2011, 7, 1175-1180.

(42) Dewar, M. J. S. The Molecular Orbital Theory of Organic Chemistry; McGraw-Hill: New York, 1969.

(43) Morita, Y.; Suzuki, S.; Sato, K; Takui, T. Synthetic Organic Spin Chemistry for Structurally Well-Defined Open-Shell Graphene Fragments. Nat. Chem. 2011, 3, 197-204.

(44) Ferrari, A. C.; Meyer, J. C.; Scardaci, V.; Casiraghi, C.; Lazzeri, M.; Mauri, F.; Piscanec, S.; Jiang, D.; Novoselov, K. S.; Roth, S.; et al. Raman Spectrum of Graphene and Graphene Layers. Phys. Rev. Lett. 2006, 97, 187401-1-187401-4.

(45) Malard, L. M.; Pimenta, M. A.; Dresselhaus, G; Dresselhaus, M. S. Raman Spectroscopy in Graphene. Phys. Rep. 2009, 473, 51-87.

(46) Delgado, J. C.; Herrera, J. M. R.; Jia, X.; Cullen, D. A.; Muramatsu, H.; Kim, Y. A.; Hayashi, T.; Ren, Z.; Smith, D. J.; Okuno, Y.; et al. Bulk Production of a New Form of $\mathrm{sp}^{2}$ Carbon: Crystalline Graphene Nanoribbons. Nano Lett 2008, 8, 2773-2778.

(47) Ryu, S.; Han, M. Y.; Maultzsch, J.; Heinz, T. F.; Kim, P.; Steigerwald, M; Brus, L. E. Reversible Basal Plane Hydrogenation of Graphene. Nano Lett. 2008, 8, 4597-4602.

(48) Luo, Z.; Yu, T.; Kim, K.-j.; Ni, Z.; You, Y.; Lim, S.; Shen, Z.; Wang, S; Lin, J. Thickness-Dependent Reversible Hydrogenation of Graphene Layers. ACS Nano 2009, 3, 1781-1788.

(49) Koehler, F. M.; Jacobsen, A.; Ensslin, K.; Stampfer, C; Stark, W. J. Selective Chemical Modification of Graphene Surfaces: Distinction Between Single- and Bilayer Graphene. Small 2010, 6, 1125-1130.

(50) Sharma, R.; Baik, J. H.; Perera, C. J; Strano, M. S. Anomalously Large Reactivity of Single Graphene Layers and Edges toward Electron Transfer Chemistries. Nano Lett. 2010, 10, 398-405.

(51) Mohiuddin, T. M. G.; Lombardo, A.; Nair, R. R.; Bonetti, A.; Savini, G.; Jalil, R; Bonini, N.; Basko, D. M.; Galiotis, C.; Marzari, N.; et al. Uniaxial Strain in Graphene by Raman Spectroscopy: G Peak Splitting, Gruneisen Parameters, and Sample Orientation. Phys. Rev. B 2009, 79, 205443.

(52) Robinson, J. A.; Wetherington, M.; Tedesco, J. L.; Campbell, P. M.; Weng, X.; Stitt, J.; Fanton, M. A.; Frantz, E.; Snyder, D.; VanMil, B. L.; et al. Correlating Raman Spectral Signatures with Carrier Mobility in Epitaxial Graphene: A Guide to Achieving High Mobility on the Wafer Scale. Nano Lett. 2009, 9, 2873-2876.

(53) Ramesh, P.; Itkis, M. E.; Bekyarova, E.; Wang, F.; Niyogi, S.; Chi, X.; Berger, C.; de Heer, W. A; Haddon, R. C. Electro-oxidized Epitaxial Graphene Channel Field-Effect Transistors with Single-Walled Carbon Nanotube Thin Film Gate Electrode. J. Am. Chem. Soc. 2010, 132, 14429-14436.

(54) Bolotin, K. I.; Sikes, K. J.; Jiang, Z.; Klima, M.; Fudenberg, G.; Hone, J.; Kim, P; Stormer, H. L. Ultrahigh Electron Mobility in Suspended Graphene. Solid State Commun. 2008, 146, 351-355.

(55) Chen, J. H.; Jang, C.; Xiao, S. D.; Ishigami, M; Fuhrer, M. S. Intrinsic and Extrinsic Performance Limits of Graphene Devices on $\mathrm{SiO}_{2}$. Nat. Nanotechnol. 2008, 3, 206-209.

(56) Bolen, M. L.; Shen, T.; Gu, J. J.; Colby, R.; Stach, E. A.; Ye, P. D; Capano, M. A. Empirical Study of Hall Bars on Few-Layer Graphene on C-Face 4H-SiC. J. Electron. Mater. 2010, 39, 2696-2701.
(57) Mathieu, C.; Barrett, N.; Rault, J.; Mi, Y. Y.; Zhang, B.; de Heer, W. A.; Berger, C.; Conrad, E. H.Renault, O. Microscopic Correlation Between Chemical and Electronic States in Epitaxial Graphene on SiC(0001). Phys. Rev. B 2011, 83, 235436.

(58) Horcas, I.; Fernandez, R.; Gomez-Rodriguez, J. M.; Colchero, J.; Gomez-Herrero, J; Baro, A. M. WSXM: A Software for Scanning Probe Microscopy and a Tool for Nanotechnology. Rev. Sci. Instrum. 2007, 78, 013705.

(59) Mallet, P.; Varchon, F.; Naud, C.; Magaud, L.; Berger, C; Veuillen, J. Y. Electron States of Mono- and Bilayer Graphene on SiC Probed by Scanning-Tunneling Microscopy. Phys. Rev. B 2007, 76, 041403(R).

(60) Rutter, G. M.; Crain, J. N.; Guisinger, N. P.; Li, T.; First, P. N; Stroscio, J. A. Scattering and Interference in Epitaxial Graphite. Science 2007, 317, 219-222.

(61) Castro, E. V.; Peres, N. M. R.; Stauber, T; Silva, N. A. P. LowDensity Ferromagnetism in Biased Bilayer Graphene. Phys. Rev. Lett. 2008, 100, 186803.

(62) Uchoa, B.; Kotov, V. N.; Peres, N. M. R; Castro Neto, A. H. Localized Magnetic States in Graphene. Phys. Rev. Lett. 2008, 101, 026805.

(63) Pisani, L.; Montanari, B; Harrison, N. M. A Defective Graphene Phase Predicted to be a Room Temperature Ferromagnetic Semiconductor. New J. Phys. 2008, 10, 033002.

(64) Barzola-Quiquia, J.; Yao, J. L.; Rodiger, P.; Schindler, K; Esquinazi, P. Sample Size Effects on the Transport Characteristics of Mesoscopic Graphite Samples. Phys. Status Solidi A 2008, 205, 2924-2933.

(65) Garcia, N.; Esquinazi, P.; Barzola-Quiquia, J.; Ming, B; Spoddig, D. Transition from Ohmic to Ballistic Transport in Oriented Graphite: Measurements and Numerical Simulations. Phys. Rev. B 2008, 78, 035413.

(66) Arndt, A.; Spoddig, D.; Esquinazi, P.; Barzola-Quiquia, J.; Dusari, S; Butz, T. Electric Carrier Concenrtation in Graphite: Dependence of Electrical Resistivity and Magnetoresistance on Defect Concentration. Phys. Rev. B 2009, 80, 195402.

(67) Wu, X.; Li, X.; Song, Z.; Berger, C; De Heer, W. A. Weak Antilocalization in Epitaxial Graphene: Evidence for Chiral Electrons. Phys. Rev. Lett. 2007, 98, 136801.

(68) Morozov, S. V.; Novoselov, K. S.; Katsnelson, M. I.; Schedin, F.; Ponomarenko, L. A.; Jiang, D; Geim, A. K. Strong Suppression of Weak Localization in Graphene. Phys. Rev. Lett. 2006, 97, 016801.

(69) Gonzalez, J. C.; Munoz, M.; Garcia, N.; Barzola-Quiquina, J.; Spodding, D.; Schindler, K; Esquinazi, P. Sample-Size Effects in the Magnetoresistance of Graphite. Phys. Rev. Lett. 2007, 99, 216601.

(70) Tombros, N.; Jozsa, C.; Popinciuc, M.; Jonkman, H. T; van Wees, B. J. Electronic Spin Transport and Spin Precession in Single Graphene Layers at Room Temperature. Nature 2007, 448, 571-575.

(71) Yazyev, O. V; Katsnelson, M. I. Magnetic Correlations at Graphene Edges: Basis for Novel Spintronic Devices. Phys. Rev. Lett. 2008, 100, 047209.

(72) Wolf, S. A.; Awschalom, D. D.; Buhrman, R. A.; Daughton, J. M.; von Molnar, S.; Roukes, M. L.; Chtchelkanova, A. Y; Treger, D. M. Spintronics A Spin-Based Electronics Vision for the Future. Science 2001, 294, 1488-1495.

(73) Kim, K.; Lee, Z.; Regan, W.; Kisielowski, C.; Crommie, M. F; Zettl, A. Grain Boundary Mapping in Polycrystalline Graphene. ACS Nano 2011, 5, 2142-2146.

(74) Huang, P. Y.; Ruiz-Vargas, C. S.; van der Zande, A. M.; Whitney, W. S.; Levendorf, M. P.; Kevek, J. W.; Garg, S.; Alden, J. S.; Hustedt, C. J.; Zhu, Y.; et al. Grains and Grain Boundaries in SingleLayer Graphene Atomic Patchwork Quilts. Nature 2011, 469, 389-393.

(75) Yazyev, O. V; Louie, S. G. Electronic Transport in Polycrystalline Graphene. Nat. Mater. 2010, 9, 806-809.

(76) Odaka, S.; Miyazaki, H.; Li, S. L.; Kanda, A.; Morita, K.; Tanaka, S.; Miyata, Y.; Kataura, H.; Tsukagoshi, K; Aoyagi, Y. Anisotropic Transport in Graphene on SiC Substrate with Periodic Nanofacets. Appl. Phys. Lett. 2010, 96, 062111.

(77) Blake, P.; Yang, R.; Morozov, S. V.; Schedin, F.; Ponomarenko, L. A.; Zhukov, A. A.; Nair, R. R.; Grigorieva, I. V.; Novoselov, K. S; Geim, 
A. K. Influence of Metal Contacts and Charge Inhomogeneity on Transport Properties of Graphene Near the Neutrality Point. Solid State Commun. 2009, 149, 1068-1071.

(78) Hossain, M. Z.; Walsh, M.; Hersam, M. C. Scanning Tunneling Microscopy, Spectroscopy, and Nanolithography of Epitaxial Graphene Chemically Modified with Aryl Moieties. J. Am. Chem. Soc. 2010, 132, 15399-15403. 